\title{
Antenna Orientation Optimization for Minimum-Energy Multicast Tree Construction in Wireless Ad Hoc Networks with Directional Antennas
}

\author{
Song Guo and Oliver Yang \\ School of Information Technology and Engineering \\ University of Ottawa \\ Ottawa, Ontario, Canada, K1N 6N5 \\ \{sguo, yang\}@site.uottawa.ca
}

\begin{abstract}
Energy conservation is a critical issue in wireless ad hoc networks since batteries are the only energy source to power the nodes. One major metric for energy conservation is to route a communication session along the routes which require the lowest total energy consumption when all nodes are equipped with a finite and nonrenewable amount of energy. To explore advantages offered by the use of directional antennas, we consider the case of source initiated multicast traffic in wireless ad hoc networks that use switched antennas and have limited energy resources. In this paper, we present a constraint formulation in terms of mixed integer linear programming, which can be used for an optimal solution of the minimum-energy multicast problem in wireless ad hoc networks with directional antennas. The optimal solutions can be used to assess the performance of heuristic algorithms for mobile networks by running them at discrete time instances.
\end{abstract}

\section{Categories and Subject Descriptors}

C.2.1 [Computer-Communication Networks]: Network Architecture and Design - Wireless Communication; G.2.2 [Discrete Mathematics]: Graph Theory - trees; G.1.6 [Numerical Analysis]: Optimization - integer programming.

\section{General Terms}

Algorithms, Performance, Experimentation, Theory.

\section{Keywords}

Wireless Ad Hoc Networks, Directional Antenna, MinimumEnergy Routing, Multicast Tree, Integer Programming.

\section{INTRODUCTION}

Ad hoc wireless networks are expected to be deployed in a wide variety of civil and military applications. The communicating nodes might be distributed randomly and are assumed to have the

Permission to make digital or hard copies of all or part of this work for personal or classroom use is granted without fee provided that copies are not made or distributed for profit or commercial advantage and that copies bear this notice and the full citation on the first page. To copy otherwise, or republish, to post on servers or to redistribute to lists, requires prior specific permission and/or a fee.

MobiHOC'04, May 24-26, 2004, Roppongi, Japan.

Copyright 2004 ACM 1-58113-849-0/04/0005 ...\$5.00. capacity of packet forwarding to communicate with each other over a shared and limited radio channel. Building such networks poses a significant technical challenge because of the constraints imposed by the characteristics of the ad hoc networks. One important constraint is the scarce power resource if the nodes are operated by batteries. Thus, for increasing longevity of such networks, it is imperative that we find ways of either increasing battery power or alternatively optimizing the use of the battery power via energy-efficient algorithms and mechanisms. Obviously, the first solution is technology dependent, and we focus on the second one that is of much interest in network research.

Recently, the problem of minimizing the energy consumption of wireless ad hoc networks has been studied comprehensively. This problem is referred to as the Minimum-Energy Routing (MER) [1] [2], which can be classified into two categories: minimumenergy unicast routing $[4,5,6,12,13,14,15]$ and minimumenergy broadcast or multicast routing $[3,16,17,18,19,20]$. The minimum-energy unicast routing is essentially a shortest directed path problem based on various power cost function. However, for broadcast applications, and in general multicast applications, the minimum-energy routing is far more challenging, which has been shown to be NP-complete [7]. Since the MER problem is hard, several heuristic algorithms for building a source based energyefficient broadcast/multicast tree have been developed. A straight greedy approach is the use of broadcast trees that consist of the best unicast paths to each individual destination from the source node (broadcast session initiator). This heuristic first applies the Dijkstra's algorithm to obtain a Shortest Path Tree (SPT), and then to orient it as a tree rooted at the source node. Similarly the Minimum Spanning Tree (MST) heuristic first applies the Prim's algorithm to obtain a MST, and then to orient it as a tree rooted at the source node. In [3, 20], another heuristic called Multicast Incremental Power (MIP) was presented. It exploits the wireless multicast advantage property ${ }^{1}$ in the formation of the multicast trees, and thus provides better performance than the greedy algorithms SPT and MST.

\footnotetext{
1 The "wireless multicast advantage" property, originally introduced in [3], means that all nodes within communication range of a transmitting node can receive a multicast message with only one transmission if they all use omni-directional antennas.
} 
It has been shown earlier that the use of directional antenna in the context of wireless ad hoc networks can largely reduce the radio interference, thereby improving the utilization of wireless medium and consequently the network performance. Some papers $[21,22]$ suggest the use of multiple directional antennas per node (or multiple beam antennas) in order to increase the throughput of 802.11 media access control protocol [23]. In [24], the author explores the use of beam forming antennas in order to improve both throughput and delay in ad-hoc networks. Another paper [25] has suggested the use of multiple directional antennas to reduce the routing overhead of on-demand routing protocols for ad-hoc networks like DSR [26] and AODV [27]. Over the past few years, energy efficient communication in wireless ad hoc networks with directional antennas has received more and more attention. An energy-efficient routing and scheduling algorithm [11] was used to coordinate transmissions in ad hoc networks where each node has a single directional antenna. A recent paper [10] extended the results in $[3,20]$ and induced two protocols Reduced-Beamwidth MIP (RB-MIP) and Directional-MIP (D-MIP) that exploit the use of directional antennas for multicasting in wireless networks.

In our earlier study [8], we introduced a new concept called virtual relay that allows the construction of a minimum-energy multicast tree to be equivalently mapped to the construction of a minimum-energy virtual relay tree. This results in the minimumenergy multicast (MEM) problem modeled as a mixed integer linear programming (MILP) problem. The optimal solution would therefore be obtained using an MILP solver available in the public domain. This paper extends the work of [8], not only by presenting a more general study from broadcast to multicast case, but also by improving our analytical model with the use of directional antennas. We formulate a generalized antenna orientation optimization and minimum-energy multicast problem that includes the problems addressed in [8] as special cases.

The remaining of this paper is organized as follows. In Section 2, we analyze the challenges for minimum-energy broadcast and multicast routing in a wireless environment. Section 3 gives a definition of minimum energy multicast tree in the context of directional antenna applications as the basis of the formulation. From Section 4 to Section 8, we construct linear constraints for Problem MEM systematically, complete formulation of the problem in a form of Integer Linear Programming, and prove that it produces the optimal solutions. Finally in Section 9, we summarize our results and point out several future research problems. For the convenience of the readers, the notations used in this paper are listed in the Appendix.

\section{ANTENNA MODEL}

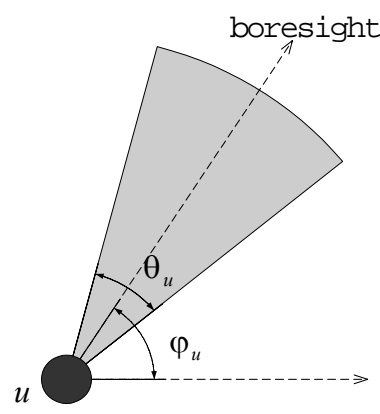

An ad-hoc wireless network consists of a fixed number of nodes, which are randomly distributed over a two-dimensional plane. Each node is equipped with a directional antenna, which permits energy savings by concentrating transmission energy where it is needed.

We use an idealized directional antenna propagation model as shown in Fig 1, where the antenna orientation $\varphi_{v}\left(0 \leq \varphi_{v}<2 \pi\right)$ of node $v$ is defined as the angle measured counter-clockwise from the horizontal axis to the antenna boresight, and the antenna directionality is specified as the angle of beamwidth $\theta_{v}\left(0 \leq \theta_{v}<\right.$ $2 \pi)$. Based on this model, antenna can be loosely classified into omni-directional, modestly directional (switched antenna), and highly directional (adaptive antenna), which are listed in Table 1. There have been a number of antenna products in each of the category.

Table 1. Antenna classification

\begin{tabular}{llll}
\hline & $\begin{array}{l}\text { Omni- } \\
\text { directional }\end{array}$ & $\begin{array}{l}\text { Modestly } \\
\text { directional }\end{array}$ & $\begin{array}{l}\text { Highly } \\
\text { directional }\end{array}$ \\
\hline $\begin{array}{l}\text { Antenna } \\
\text { Directionality }\end{array}$ & $\begin{array}{l}\text { fixed } \\
\text { beamwidth }\end{array}$ & $\begin{array}{l}\text { fixed } \\
\text { beamwidth }\end{array}$ & $\begin{array}{l}\text { variable } \\
\text { beamwidth }\end{array}$ \\
$\begin{array}{l}\text { Antenna } \\
\text { Orientation }\end{array}$ & unsteerable & steerable & steerable \\
\hline
\end{tabular}

When considering omni-directional antennas and uniform propagation condition, we observe that all nodes within the communication range of a transmitting node can receive its transmission, and the received signal power varies as $r^{-\alpha}$, where $r$ $(r>1)$ is the distance to the sender, and $\alpha$ is a parameter that typically takes on a value between 2 and 4, depending on the characteristics of the communication medium. Based on this model, the transmitted power required to support a link between two nodes separated by range $r$ is proportional to $r^{\alpha}$. Without loss of generality, all receivers have the same power threshold for signal detection, which are typically normalized to one, resulting in $p_{v u}=r_{v u}^{\alpha}$, where $r_{v u}$ is the distance between node $v$ and node $u$, and $p_{v u}$ represents the power needed for link between node $v$ and node $u$. For our directional antenna propagation model, we further assume that for any node $v$, all of the transmitted energy is concentrated uniformly in a beamwidth, ignoring the possibility of sidelobe interference. Then, the transmission power needed by node $v$ to transmit to node $u$ in its antenna beam using beamwidth $\theta_{v}$ is

$$
p_{v u}=\frac{\theta_{v}}{2 \pi} r_{v u}^{\alpha}
$$

Consequently, the use of narrow beams allows energy saving for a given communication range or extends the antenna range for a given transmission power level when compared to the use of omnidirectional antennas. On the other hand, only the nodes located within the transmitting node's antenna beam can receive the signal, thus possibly diminishing the effect of the wireless multicast advantage. We only focus on the modestly directional antenna in this paper. All the assumptions through the whole paper are summarized below.

Beamwidth of each antenna cannot be adjusted, i.e., $\theta_{v}$ is fixed for any node $v$.

Figure 1. Directional antenna propagation model. 
Orientation of each antenna can be shifted to any desired direction to provide connectivity to a subset of the nodes that are within communication range. A single antenna beam is provided for each session in which a node participates.

Each node knows the precise locations of its potential neighbors.

\section{MINIMUM ENERGY MULTICAST TREE}

Let us model the network by a simple directed graph $G(N, A, p)$, where $N$ is a finite node set, $|N|=n$, and $A$ is an arc set corresponding to the unidirectional wireless communication links. The arc weight function $p: A \rightarrow R^{+}$assigns power to each arc, where $R^{+}$denotes the positive real number set. That is, for each $\operatorname{arc}(v, u), p_{v u}$ is the power needed for the link from node $v$ to node $u$. We assume that any node $v \in N$ can choose its power level, not to exceed some maximum value $p^{v}{ }_{\text {max }}$. Any directed arc $(v, u) \in A$ if and only if $p_{v u} \leq p^{v}{ }_{\max }$.

We consider a source-initiated multicast in wireless ad-hoc networks. Any node is permitted to initiate multicast sessions. Multicast requests and session durations are generated randomly at the network nodes. The set of multicast group members $M(|M|$ $=m$ ) and other relay nodes that support a multicast session are referred to as a multicast tree. We assume that no power expenditure is involved in signal reception and processing activities. Thus the total power is expended completely on transmission at each node in the tree. Obviously, leaf nodes do not contribute to this quantity because they do not relay traffic to any other nodes. Hence, we evaluate performance in terms of total RF power from all transmitting nodes required to maintain the tree.

Any multicast tree is a rooted tree. We define a rooted tree as a directed acyclic graph with a source node $s$ called root with no incoming arcs, and all its other nodes with exactly one incoming arc. A property of rooted tree is that for any node $u$ in the tree, there exists a single directed path from $s$ to $u$ in the tree. A node with no out-going arcs is called a leaf node, and all other nodes are internal nodes, or relay nodes. Note that the relay nodes may be multicast members or may not, and the antenna beam of a relay node should cover all its children. Therefore, the minimumenergy multicast problem is to find a multicast tree with the minimum power consumption. Doing so involves the choice of transmission power level, relay nodes, and antenna orientation.

Formally, a multicast tree is modeled by a node-weighted tree $T_{s}\left(N^{\prime}, A^{\prime}, q\right)$ rooted at a source node $s$, with a multicast node set $N^{\prime}$ $\subseteq N$, an arc set $A^{\prime} \subseteq A$, and a node weight function defined as $q$ : $N^{\prime} \rightarrow R^{+} \cup\{0\}$. That is, for each node $v$ in $N^{\prime}, q_{v}$ is the transmission power of the node $v$ required by the multicast tree $T_{s}$. We define $T_{s}\left(N^{\prime}, A^{\prime}, q\right)$ to be a multicast tree of $G(N, A)$ rooted at $s$ if and only if the following properties are satisfied.

1) RTP (Rooted Tree Property): this property requires $T_{s}$ can span all the multicast members from node $s\left(M \subseteq N^{\prime}\right)$;

2) WMAP (Wireless Multicast Advantage Property): this property requires the node weight function to satisfy:

$$
q_{u}=\left\{\begin{array}{cl}
0, & u \text { is leaf node } \\
\underset{(u, v) \in A^{\prime}}{\operatorname{Max}} p_{u v}, & u \text { is internal node }
\end{array}\right.
$$

3) AOP (Antenna Orientation Property): this property requires node $u$ must locate within the antenna beam of node $v$, for any $(v, u) \in A^{\prime}$.

\section{FORMULATION MODEL}

This is the first time that an accurate definition of multicast tree is given in the context of directional antenna applications, upon which Problem MEM shall be formulated as a mixed integer linear programming (MILP) model. The main idea is to extract a sub-graph $T_{s}{ }^{*}$ from the original graph $G$, such that $T_{s}{ }^{*}$ is a multicast tree with minimum energy consumption. In order to formulate the problem, we define the following variables:

(i) $\quad Z_{v u}$ is a binary decision variable which is equal to one if the $\operatorname{arc}(v, u)$ is in the sub-graph $T_{s}^{*}$ of $G$, and zero otherwise;

(ii) $\quad P_{v}$ is a nonnegative continuous variable which represents the transmission power of the node $v$ required by the multicast tree $T_{s}^{*}$;

(iii) $F_{v u}$ is a nonnegative continuous variable, which represents the amount of flow going through $\operatorname{arc}(v, u)$;

(iv) $\quad c_{v u}$ is a binary variable which is equal to one if node $u$ is covered by the antenna beam of node $v$, and zero otherwise.

We shall prove that if $(x)^{*}$ is the optimal solution of variable $x$ obtained from this MILP model, then the graph $T_{s}^{*}\left(N^{\prime}, A^{\prime}, q\right)$ is the optimal tree associated with this solution. In this graph, $N^{\prime}=\{u \mid$ $\exists(v, u) \in A^{\prime}$ or $\left.(u, v) \in A^{\prime}\right\}$ is its arc set, $A^{\prime}=\left\{(v, u) \mid Z_{v u}^{*}=1\right\}$ is its arc set, and $q: A^{\prime} \rightarrow R^{+} \cup\{0\}$ is a nonnegative weight function defined as $q_{u}=P_{u}^{*}$. In other words, $T_{s}^{*}\left(N^{\prime}, A^{\prime}, q\right)$ is a multicast tree of $G$ with minimum energy consumption. Finally, the Problem MEM can be formulated to minimize total power from all transmitting nodes subject to the constraints that $T_{s}^{*}$ satisfies RTP, WMAP, and AOP. We then have the following formulation for the Problem MEM as shown below.

Inputs: $G(N, A, p), M$, and $s$

Objective function: $\sum_{v \in N} P_{v}$

Constraints: RTP, WMAP, and AOP

Output: $T_{s}^{*}\left(N^{\prime}, A^{\prime}, q\right)$

Example 1

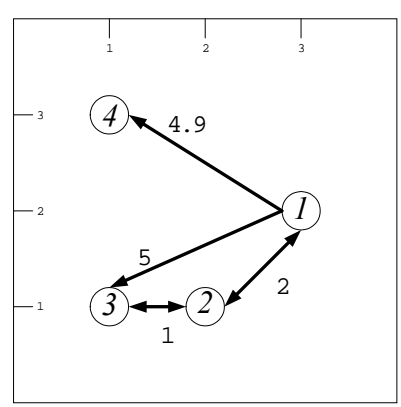

Figure 2. Example 4-node network G4: multicast group is $\{1$, $2,3\}$ and node 1 is the source

A generic example of a 4-node network $G^{4}$ that we consider is shown in Figure 2. The weight for each arc represents the power required transmitting packets on it. $G^{4}$ is an asymmetric directed graph. For example, the double arrow arc $(1,2)$ indicates that 
node 1 and node 2 can reach each other, while the unidirectional arc $(1,4)$ indicates that only node 1 can reach node 4 since node 4 may not have enough power to reach node 1 . We assume the channel loss exponent $\alpha=2$, then we can obtain $\angle 123=3 \pi / 4$. We assume each node has a fixed antenna beamwidth $\pi$. The objective function of $G^{4}$ is therefore:

$$
\text { minimize: } P_{1}+P_{2}+P_{3}+P_{4}
$$

\section{LINEAR CONSTRAINTS FOR RTP}

We want to provide a set of constraints that would guarantee that $T_{s}^{*}\left(N^{\prime}, A^{\prime}, q\right)$ obtained from the formulation satisfies the rooted tree property. It can be characterized that $T_{s}^{*}\left(N^{\prime}, A^{\prime}, q\right)$ is a rooted tree spanning all the multicast members, i.e., $M \subseteq N^{\prime}$, by the following properties:

RTP (a): $\quad$ Every node $u, u \in N^{\prime} \backslash\{s\}$, has exactly one incoming arc, and node $s$ has no incoming arcs;

RTP (b): $\quad T_{s}^{*}\left(N^{\prime}, A^{\prime}, q\right)$ does not contain cycles.

The construction and interpretation of the linear constraints for these two properties are elaborated in the following lemmas.

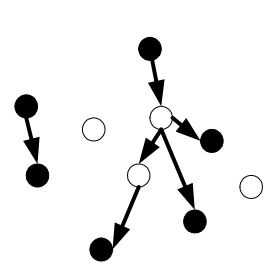

(a)

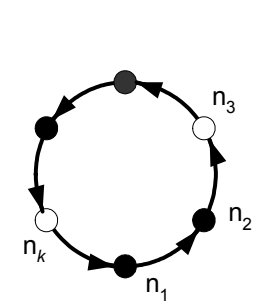

(b)

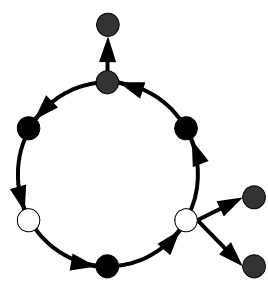

(c)
Figure 3. Illustration of constraints (a) any non-multicast member in $V^{*}$ must have exactly one incoming arc, (b) a connected component of $V^{*}$ may be a simple cycle, (c) a cycle with sub tree leaving out of it. (Solid nodes indicate multicast members, and hollow nodes indicate non-multicast members.)

Lemma 1. $T_{s}^{*}\left(N^{\prime}, A^{\prime}, q\right)$ is a directed graph in which node $s$ has no incoming arcs, and each other node has exactly one incoming arc, provided Problem MEM satisfies the following constraints:

$$
\begin{aligned}
& \sum_{v \in N} Z_{v s}=0 ; \\
& \sum_{v \in N} Z_{v u}=1 ; \forall u \in M \backslash\{s\} \\
& \sum_{v \in N} Z_{v u} \leq 1 ; \forall u \in N \backslash M \\
& \sum_{v \in N} Z_{u v} \leq(n-1) \sum_{v \in N} Z_{v u} ; \forall u \in N \backslash M
\end{aligned}
$$

Proof: Note that $\Sigma_{v \in N} Z_{v u}^{*}$ and $\Sigma_{v \in N} Z_{u v}^{*}$ are the in-degree and outdegree of node $u$ in $T_{s}^{*}$ respectively. Therefore, the root node $s$ and the other multicast members satisfy this statement directly from the constraints (3) and (4) respectively. It remains to prove that any non-multicast member in $T_{s}^{*}$ supporting the multicast communications must have exactly one incoming arc.

Assume $u \in N^{\prime}$ is a non-multicast member in $T_{s}{ }^{*}$, indicated by a hollow node in Fig. 3, its incoming degree must be 1 or 0 from constraints (5). If $\Sigma_{v \in N} Z_{v u}^{*}=0$, from constraints (6), it follows that $\Sigma_{v \in N} Z_{u v}^{*}=0$. That means $u$ must be an isolated node as shown in Fig. 3a, thus $u \notin N^{\prime}$. This contradicts the original assumption. Therefore node $u$ has exactly one incoming arc.

Note that if $\Sigma_{v \in N} Z_{v u}^{*}=1$ for any non-multicast member $u$ in $T_{s}{ }^{*}$, constraints (6) become redundant since the out-degree of node $u$ is at most $n-1$. From constraints (3), (4), and (5), we obtain the following conclusion:

$$
\sum_{v \in N} Z_{v u} \in\{0,1\}, \forall u \in N
$$

Example 2

Referring to $G^{4}$ in Figure 2, we can now list the first set of constraints corresponding to (3) to (6) for RTP (a) as follows:

$$
\begin{aligned}
& Z_{21}=0 \\
& Z_{12}+Z_{32}=1 \\
& Z_{13}+Z_{23}=1 \\
& Z_{14} \leq 1 \\
& 3 Z_{14} \geq 0
\end{aligned}
$$

We shall see in the Lemma 2 that the introduction of variable $F_{v u}$ is to help to prevent loops in $T_{s}$, and this variable only represents fictitious flow produced by the multicast initiator $s$ going through $\operatorname{arc}(v, u)$.

Lemma 2. $V^{*}\left(N^{\prime}, A^{\prime}, q\right)$ does not contain cycles, if Problem MEM satisfies the constraints (3) - (5) and the following constraints:

$$
\begin{aligned}
& \sum_{v \in N} F_{v u}-\sum_{v \in N} F_{u v}=\sum_{v \in N} Z_{v u} ; \forall u \in N \backslash\{s\} \\
& Z_{v u} \leq F_{v u} \leq(n-1) Z_{v u} ; \forall u \in N \backslash\{s\}, v \in N
\end{aligned}
$$

Proof: From the constraints in (3), (4) and (5), it follows that the only connected components in $T_{s}^{*}$ that might contain cycles could be composed of either a simple cycle shown in Fig. $3 b$, or a simple cycle with sub tree leaving out of it as shown in Fig. 3c. We will show in the following that such topologies are not feasible for Problem MEM.

Assume that the nodes $\left(n_{1}, n_{2}, \ldots, n_{k}, n_{k+1}=n_{1}\right), k>1$, form a simple cycle in $T_{s}{ }^{*}$. Then from constraint (3), node $s$ will never be included in such a cycle. Constraint in (9) implies that $F^{*}{ }_{v u}$ could be positive if and only if $(v, u) \in A^{\prime}$. Letting $F_{n_{1} n_{2}}^{*}=f$, then from the constraints in (8) it follows that $F_{n_{r} n_{r+1}}^{*}=F_{n_{1} n_{2}}^{*}-$ $\sum_{i=1}^{r-1} Z_{n_{i} n_{i+1}}^{*}$ for $r=1, \ldots, k$. Each node $n_{r}(r=1, \ldots, k)$ is in $A^{\prime}$ as stated in the assumption, i.e., $Z_{n_{r} n_{r+1}}^{*}=1$. Therefore $F_{n_{r} n_{r+1}}^{*}=$ $F_{n_{1} n_{2}}^{*}-\sum_{i=1}^{r-1} Z_{n_{i} n_{i+1}}^{*}=f-(r-1)$ for $r=1, \ldots, k$. After substituting $F_{n_{k} n_{1}}^{*}=f-(k-1)$ into constraint (8), for $u=n_{1}$, we obtain $\sum_{v \in N} F_{v n_{1}}^{*}-\sum_{v \in N} F_{n_{1} v}^{*}=f-(k-1)-f=1-k<0$. On the other hand, $\sum_{v \in N} F_{v n_{1}}^{*}-\sum_{v \in N} F_{n_{1} v}^{*}=\sum_{v \in N} Z_{v n_{1}}^{*} \geq 0$ from constraints (7). Thus the constraints in (8) are violated, and therefore simple cycles are not possible in $T_{s}{ }^{*}$. Similar reasoning shows that the topology in Fig. 3c also violates the constraints in (8), and therefore $T_{s}^{*}$ cannot contain cycles.

Example 3 
Still referring to $G^{4}$ in Figure 2, we can list the next set of constraints corresponding to (8) and (9) for RTP (b), which is expressed as follows:

$$
\begin{aligned}
& F_{12}+F_{32}-F_{21}-F_{23}=Z_{12}+Z_{32} \\
& F_{13}+F_{23}-F_{32}=Z_{13}+Z_{23} \\
& F_{14}=Z_{14} \\
& Z_{12} \leq F_{12} \leq 3 Z_{12} \\
& Z_{32} \leq F_{32} \leq 3 Z_{32} \\
& Z_{13} \leq F_{13} \leq 3 Z_{13} \\
& Z_{23} \leq F_{23} \leq 3 Z_{23} \\
& Z_{14} \leq F_{14} \leq 3 Z_{14}
\end{aligned}
$$

\section{LINEAR CONSTRAINTS FOR WMAP}

The constraints for the wireless multicast advantage property (WMAP) reflect the condition that the power required at node $u$ is the maximum of the individual transmission power to each neighbor from $u$. The following lemma explains how the WMAP, i.e., Equation (2), can be achieved.

Lemma 3. $T_{s}^{*}\left(N^{\prime}, A^{\prime}, q\right)$ satisfies WMAP, if the formulation of Problem MEM includes the constraint (10).

$$
P_{v} \geq p_{v u} Z_{* u} ; \forall v, u \in N
$$

Proof: For any node $v$ in $T_{s}^{* u}$, if $v$ is a leaf node, i.e., $Z_{v u}^{*}=0$ for all $u \in N^{\prime}$, then $P^{*}{ }_{v} \geq p_{v u} Z_{v u}{ }_{v u}=0$; if $v$ is an internal node, then $P^{*}{ }_{v} \geq$ $p_{v u} Z_{v u}^{*}$ for all $u \in N^{\prime}$, i.e., $P^{*}{ }_{v} \geq \operatorname{Max}_{(v, u) \in A^{\prime}} p_{v u}$. The equalities are achieved in the inequations above when the summation of the variables $P_{v}$ is minimized. Thus Equation (2) must be held by $T_{s}^{*}$.

\section{Example 4}

The set of constraints for the example $G^{4}$ shown in Figure 2 corresponding to the constraints (10) for WMAP can be expressed as follows:

$$
\begin{aligned}
& P_{1} \geq 2 Z_{12} \\
& P_{1} \geq 5 Z_{13} \\
& P_{1} \geq 4.9 Z_{14} \\
& P_{2} \geq 2 Z_{21} \\
& P_{2} \geq Z_{23} \\
& P_{3} \geq Z_{32}
\end{aligned}
$$

\section{LINEAR CONSTRAINTS FOR AOP}

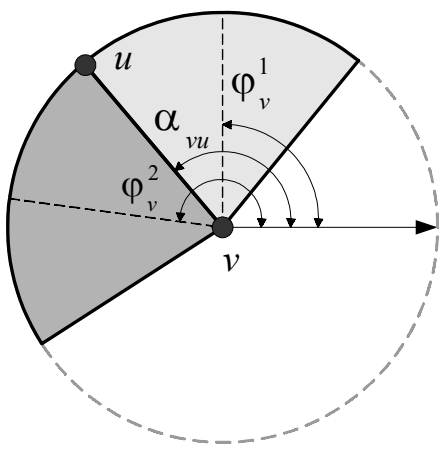

Figure 4. Antenna beam coverage range

As defined in Section 2, the antenna orientation $\left\{\varphi_{v}: v \in N\right\}$ are continuous variables $0 \leq \varphi_{v}<2 \pi$. Let $\left\{c_{v u}:(v, u) \in A\right\}$ be binary variables such that $c_{v u}=1$ if node $u$ is covered by the antenna beam of node $v$, and 0 otherwise. In other words, the wireless communication link $(v, u)$ exists if and only if $c_{v u}=1$. Before discussing the construction of linear constraints for AOP, we first investigate in more detail the relationship between the antenna orientation $\varphi_{v}$ and the existence of wireless communication links $c_{v u}$.

Let $\alpha_{v u}\left(0 \leq \alpha_{v u}<2 \pi\right)$ be the angle measured counter-clockwise from the horizontal axis to the vector $\overrightarrow{v u}$ as shown in Fig. 4. Then the angle $\alpha_{v u}(v, u \in N)$ can be obtained once their positions are given. Assume the beam width $\theta_{v}\left(0 \leq \theta_{v}<2 \pi\right)$ of each node $v$ is fixed, and each antenna beam can be pointed in any desired direction by adjusting its orientation continuously to provide connectivity to a subset of the nodes that are within its communication range. In Fig. 4, the lighter shaded area is the space covered by the antenna beam of node $v$ when it is about entering the position of node $u$ (i.e., for $v$ making contact with $u$ ), and the darker shaded area is the space just before the beam is leaving the position of node $u$ (i.e., for $v$ losing contact with $u$ ). Thus it is clear that the wireless link $(v, u)$ exists if and only if the antenna orientation $\varphi_{v}$ is bounded by the two pointing directions $\varphi^{1}{ }_{v}=\alpha_{v u}-\theta_{v} / 2$ and $\varphi^{2}{ }_{v}=\alpha_{v u}+\theta_{v} / 2$, indicated by the dotted lines as shown in Fig. 4.
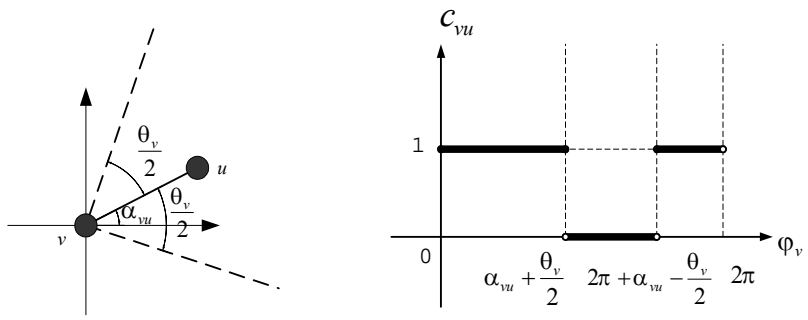

(a) $0 \leq \alpha_{v u} \leq \theta_{v} / 2$
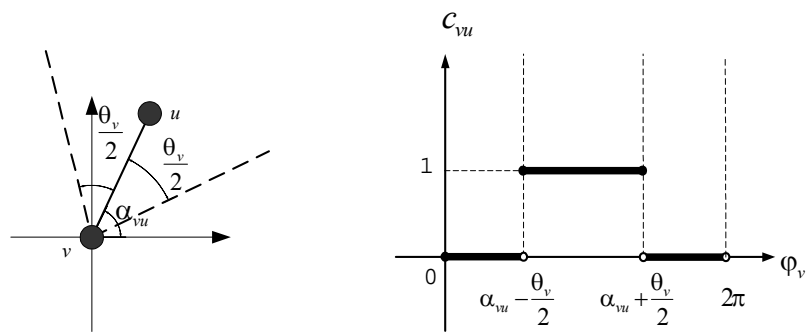

(b) $\theta_{v} / 2 \leq \alpha_{v u} \leq 2 \pi-\theta_{v} / 2$
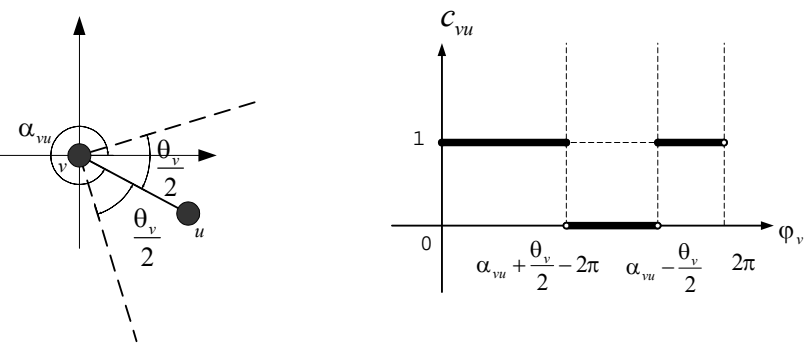

(c) $2 \pi-\theta_{v} / 2 \leq \alpha_{v u}<2 \pi$

Figure 5. Three possible value ranges of angle $\alpha_{v u}$ for the constraints between $\varphi_{v}$ and $c_{v u}$ 
Assume $0 \leq \alpha_{v u}<\theta_{v} / 2$. Then according to the definition of $c_{v u}$, the range $\left[\alpha_{v u}-\theta_{v} / 2, \alpha_{v u}+\theta_{v} / 2\right]$ of $\varphi_{v}$ shown in the left part of Fig 5a must be mapped into $\left[0, \alpha_{v u}+\theta_{v} / 2\right] \cup\left[2 \pi+\alpha_{v u}-\theta_{v} / 2,2 \pi\right)$, since we restrict variable $\varphi_{v}$ between 0 and $2 \pi$. Therefore, $c_{v u}=1$ if and only if $\varphi_{v} \in\left[0, \alpha_{v u}+\theta_{v} / 2\right] \cup\left[2 \pi+\alpha_{v u}-\theta_{v} / 2,2 \pi\right)$, which is expressed in Constraint (11) and depicted in the right part of Fig 5a. Similarly, we have Constraints (12) and (13) for $\varphi_{v}$ and $c_{v u}$ corresponding to $\alpha_{v u}$ within different ranges as shown in Figure $5 \mathrm{~b}$ and Figure $5 \mathrm{c}$ respectively.

(i) $0 \leq \alpha_{v u}<\theta_{v} / 2$ :

$c_{v v}= \begin{cases}1, & 0 \leq \varphi_{v} \leq \alpha_{v u}+\theta_{v} / 2 \text { or } 2 \pi+\alpha_{v u}-\theta_{v} / 2 \leq \varphi_{v}<2 \pi \\ 0, & \text { otherwise }\end{cases}$

(ii) $\theta_{v} / 2 \leq \alpha_{v u}<2 \pi-\theta_{v} / 2$ :

$c_{v u}=\left\{\begin{array}{lc}1, & \alpha_{v u}-\theta_{v} / 2 \leq \varphi_{v} \leq \alpha_{v u}+\theta_{v} / 2 \\ 0, & \text { otherwise }\end{array}\right.$

(iii) $2 \pi-\theta_{v} / 2 \leq \alpha_{v u}<2 \pi$ :

$c_{v u}= \begin{cases}1, & \alpha_{v u}-\theta_{v} / 2 \leq \varphi_{v}<2 \pi \text { or } 0 \leq \varphi_{v} \leq \alpha_{v u}+\theta_{v} / 2-2 \pi \\ 0, & \text { otherwise }\end{cases}$

The above Constraints (11) - (13) are obviously nonlinear. In the following three cases, we shall show that these constraints can be linearized.

Case 1: $0 \leq \alpha_{v u}<\frac{\theta_{v}}{2}$

The right part of Fig. 5a shows the Constraint (11) in a $\varphi_{v}-c_{v u}$ plane. We observe that $c_{v u}$ can be decomposed into a summation of two new binary variables $c_{v u}^{1}$ and $c^{2}{ }_{v u}$, which are defined in Equations (14) and (15).

$c_{v u}^{1}=\left\{\begin{array}{lc}1, & 0 \leq \varphi_{v} \leq \alpha_{v u}+\theta_{v} / 2 \\ 0, & \alpha_{v u}+\theta_{v} / 2<\varphi_{v}<2 \pi\end{array}\right.$

$c_{v u}^{2}=\left\{\begin{array}{cc}1, & 2 \pi+\alpha_{v u}-\theta_{v} / 2 \leq \varphi_{v}<2 \pi \\ 0, & 0 \leq \varphi_{v}<2 \pi+\alpha_{v u}-\theta_{v} / 2\end{array}\right.$

Equations (14) and (15) are depicted by thick lines in $\varphi_{v}-c^{1}{ }_{v u}$ plane and $\varphi_{v}-c^{2}{ }_{v u}$ plane as shown in Fig $6 \mathrm{a}$ and Fig $6 \mathrm{~b}$ respectively. In Fig. 6a, the points $\left(\varphi_{v}, c^{1}{ }_{v u}\right)$ that satisfy the Equation (14) must be within the shaded area between line $P_{1} P_{2}$ and line $P_{3} P_{4}$, where $P_{1}=(0,1 / 2), P_{2}=\left(\alpha_{v u}+\theta_{v} / 2,0\right), P_{3}=(2 \pi, 0)$, and $P_{4}=\left(\alpha_{v u}+\theta_{v} / 2,1\right)$. It can be clearly observed that Constraint (17) later covers the shaded area above line $P_{1} P_{2}$, and Constraint (18) covers the shaded area below line $P_{3} P_{4}$. Since $0 \leq \varphi_{v}<2 \pi$ and $c^{1}{ }_{v u} \in\{0,1\}$, the point set defined by Equation (14) and the point set defined by the constraints (17) and (18) are the same in a $\varphi_{v}-c^{1}{ }_{v u}$ plane as shown in Fig. 6a. Since $P_{1}$ may be any point between $(0,0)$ and $(0,1)$, we may choose their middle point without loss of generality.

Similarly, the points $\left(\varphi_{v}, c^{2}{ }_{v u}\right)$ defined by the Equation (15) can be rewritten in constraints (19) and (20) with the help of Fig. 6b. In summary, the nonlinear Constraint (11) is linearized using Constraints (16) to (20).

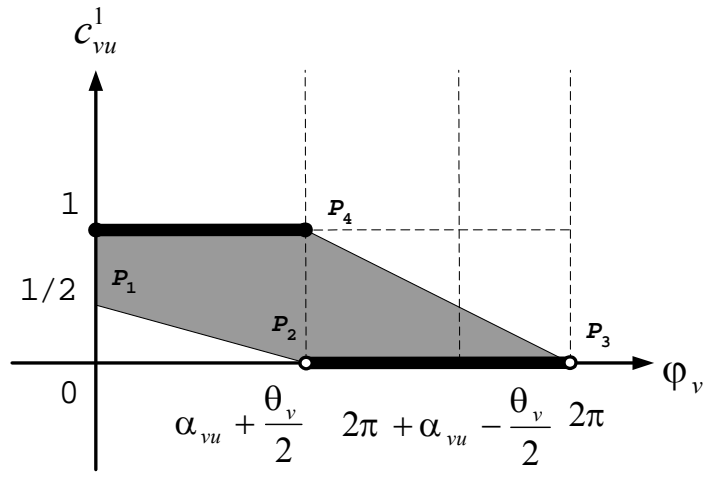

(a) linear constraint between $\varphi_{v}$ and $c^{1}{ }_{v u}$

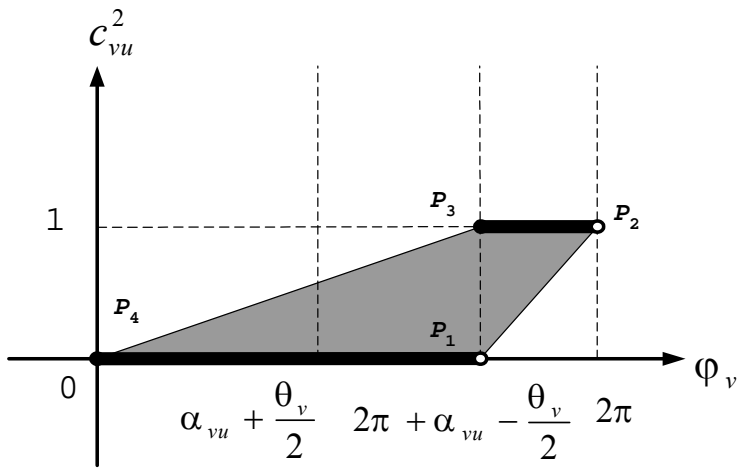

(b) linear constraint between $\varphi_{v}$ and $c_{v u}^{2}$

Figure 6. Illustration of constraint linearization $\left(0 \leq \alpha_{v u}<\theta_{v} / 2\right)$

$$
\begin{aligned}
& c_{v u}=c_{v u}^{1}+c_{v u,}^{2} 0 \leq \alpha_{v u}<\frac{\theta_{v}}{2} \\
& c_{v u}^{1}>-\frac{1}{2 \alpha_{v u}+\theta_{v}} \varphi_{v}+\frac{1}{2} \\
& c_{v u}^{1} \leq-\frac{2}{4 \pi-2 \alpha_{v u}-\theta_{v}} \varphi_{v}+\frac{4 \pi}{4 \pi-2 \alpha_{v u}-\theta_{v}} \\
& c_{v u}^{2}>\frac{2}{\theta_{v}-2 \alpha_{v u}} \varphi_{v}-\frac{4 \pi+2 \alpha_{v u}-\theta_{v}}{\theta_{v}-2 \alpha_{v u}} \\
& c_{v u}^{2} \leq \frac{2}{4 \pi+2 \alpha_{v u}-\theta_{v}} \varphi_{v}
\end{aligned}
$$

Case 2: $\frac{\theta_{v}}{2} \leq \alpha_{v u}<2 \pi-\frac{\theta_{v}}{2}$

In this case, $c_{v u}$ can be decomposed into another linear combination of binary variables $c^{1}{ }_{v u}$ and $c^{2}{ }_{v u}$, given in Equation (21) and (22). Following a similar step as above and the help of Fig. 7, we linearize Constraint (12) into Constraints (23) to (27).

$$
\begin{aligned}
& c_{v u}^{1}= \begin{cases}1, & \alpha_{v u}-\theta_{v} / 2 \leq \varphi_{v}<2 \pi \\
0, & 0 \leq \varphi_{v}<\alpha_{v u}-\theta_{v} / 2\end{cases} \\
& c_{v u}^{2}= \begin{cases}1, & 0 \leq \varphi_{v} \leq \alpha_{v u}+\theta_{v} / 2 \\
0, & \alpha_{v u}+\theta_{v} / 2<\varphi_{v}<2 \pi\end{cases} \\
& c_{v u}=c_{v u}^{1}+c_{v u}^{2}-1, \frac{\theta_{v}}{2} \leq \alpha_{v u}<2 \pi-\frac{\theta_{v}}{2}
\end{aligned}
$$




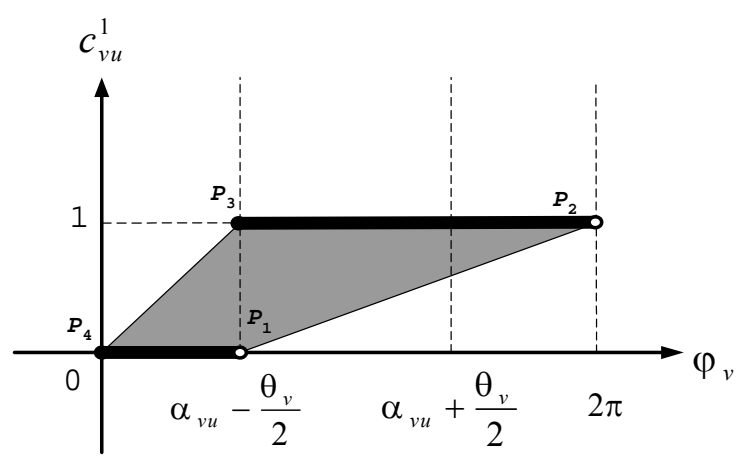

(a) linear constraint between $\varphi_{v}$ and $c^{1}{ }_{v u}$

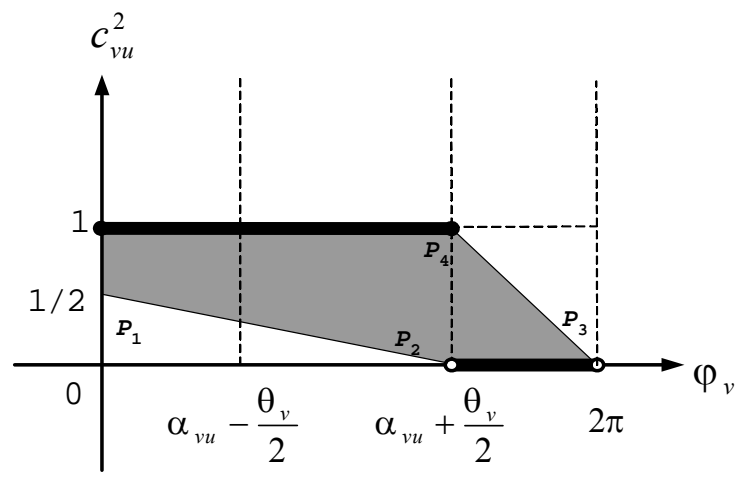

(b) linear constraint between $\varphi_{v}$ and $c^{2}{ }_{v u}$

Figure 7. Illustration of constraint linearization

$$
\left(\theta_{v} / 2 \leq \alpha_{v u}<2 \pi-\theta_{v} / 2\right)
$$

$c_{v u}^{1}>\frac{2}{4 \pi-2 \alpha_{v u}+\theta_{v}} \varphi_{v}-\frac{2 \alpha_{v u}-\theta_{v}}{4 \pi-2 \alpha_{v u}+\theta_{v}}$

$c_{v u}^{1} \leq \frac{2}{2 \alpha_{v u}-\theta_{v}} \varphi_{v}$

$c_{v u}^{2}>-\frac{2}{2 \alpha_{v u}+\theta_{v}} \varphi_{v}+\frac{1}{2}$

$c_{v u}^{2} \leq-\frac{2}{4 \pi-2 \alpha_{v u}-\theta_{v}} \varphi_{v}+\frac{4 \pi}{4 \pi-2 \alpha_{v u}-\theta_{v}}$

Case $3: 2 \pi-\frac{\theta_{v}}{2} \leq \alpha_{v u}<2 \pi$

Very similar to Case 1, linear constraints (30) to (34) illustrated in Fig. 8 can be obtained for Equation (13).

$$
\begin{aligned}
& c_{v u}^{1}= \begin{cases}1, & 0 \leq \varphi_{v} \leq \alpha_{v u}+\theta_{v} / 2-2 \pi \\
0, & \alpha_{v u}+\theta_{v} / 2-2 \pi<\varphi_{v}<2 \pi\end{cases} \\
& c_{v u}^{2}= \begin{cases}1, & \alpha_{v u}-\theta_{v} / 2 \leq \varphi_{v}<2 \pi \\
0, & 0 \leq \varphi_{v}<\alpha_{v u}-\theta_{v} / 2\end{cases} \\
& c_{v u}=c_{v u}^{1}+c_{v u}^{2}, 2 \pi-\frac{\theta_{v}}{2} \leq \alpha_{v u}<2 \pi \\
& c_{v u}^{1}>-\frac{1}{2 \alpha_{v u}+\theta_{v}-4 \pi} \varphi_{v}+\frac{1}{2}
\end{aligned}
$$

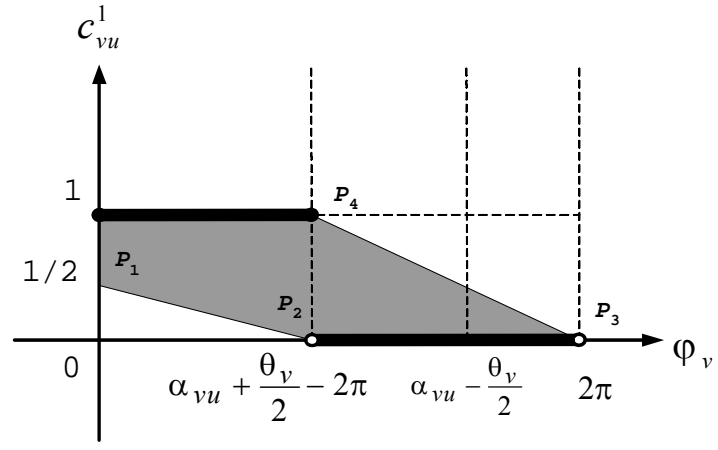

(a) linear constraint between $\varphi_{v}$ and $c^{1}{ }_{v u}$

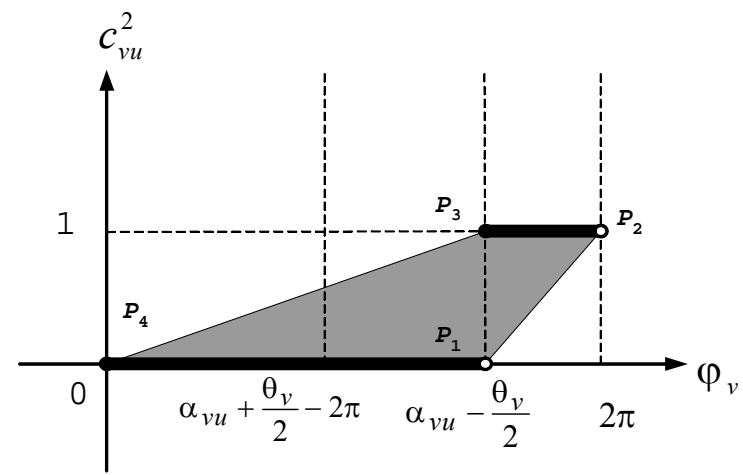

(b) linear constraint between $\varphi_{v}$ and $c_{v u}^{2}$

Figure 8. Illustration of constraint linearization

$$
\left(2 \pi-\theta_{v} / 2 \leq \alpha_{v u}<2 \pi\right)
$$

$c_{v u}^{1} \leq-\frac{2}{8 \pi-2 \alpha_{v u}-\theta_{v}} \varphi_{v}+\frac{4 \pi}{8 \pi-2 \alpha_{v u}-\theta_{v}}$

$c_{v u}^{2}>\frac{2}{4 \pi+\theta_{v}-2 \alpha_{v u}} \varphi_{v}-\frac{2 \alpha_{v u}-\theta_{v}}{4 \pi+\theta_{v}-2 \alpha_{v u}}$

$c_{v u}^{2} \leq \frac{2}{2 \alpha_{v u}-\theta_{v}} \varphi_{v}$

So far, the linear constraints have been completely constructed to characterize the relation between antenna orientation $\varphi_{v}$ and the existence of wireless communication links $c_{v u}$, upon which the Antenna Orientation Property (AOP) can be easily achieved by the following lemma.

Lemma 4. $T_{s}^{*}\left(N^{\prime}, A^{\prime}, q\right)$ satisfies AOP, if the formulation of Problem MEM includes the following constraints:

$$
Z_{v u} \leq c_{v u} ; \forall v, u \in N
$$

Proof: $\operatorname{Arc}(v, u)$ exists in $T_{s}^{*}$, i.e. $Z_{v u}^{*}=1$, only if node $u$ locates within the antenna beam of node $v$, i.e. $c_{v u}^{*}=1$. This is equivalent to Constraint (35)

\section{Example 5}

Referring the example of the 4-node network shown in Fig. 2, we consider the additional constraints imposed on variables $Z_{v u}$ once taking into account AOP in a context of directional antenna applications. As we assumed before, each node has a fixed 
beamwidth $\pi$. We only discuss the linear constraint formulation about variables $Z_{12}$ and $Z_{21}$ as examples.

For constraints of $Z_{12}$, since $\alpha_{12}=5 \pi / 4, \theta_{1}=\pi$, and $\theta_{1} / 2 \leq \alpha_{12}<$ $2 \pi-\theta_{1} / 2$, this belongs to Case 2 . For constraints of $Z_{21}$, since $\alpha_{21}$ $=\pi / 4, \theta_{2}=\pi$, and $0 \leq \alpha_{21}<\theta_{2} / 2$, it belongs to Case 1 . We list the constraints for AOP corresponding to $Z_{12}$ and $Z_{21}$ expressed as follows:

$$
\begin{array}{rlrl}
Z_{12} & \leq c_{12}^{1}+c_{12}^{2}-1 & Z_{21} & \leq c_{21}^{1}+c_{21}^{2} \\
c_{12}^{1} & >4 \varphi_{1} / 5 \pi-3 / 5 & & c_{21}^{1}>-2 \varphi_{2} / 3 \pi+1 / 2 \\
c_{12}^{1} & \leq 4 \varphi_{1} / 3 \pi & & c_{21}^{1} \leq-4 \varphi_{2} / 5 \pi+8 / 5 \\
c_{12}^{2} & >-4 \varphi_{1} / 7 \pi+1 / 2 & & c_{21}^{2}>4 \varphi_{2} / \pi-7 \\
c_{12}^{2} & \leq-4 \varphi_{1} / \pi+8 & c_{21}^{2} \leq 4 \varphi_{2} / 7 \pi
\end{array}
$$

\section{PROBLEM MEM FORMULATION}

Our previous derivation on the linear constraints can now help us to rewrite the problem formulation in Section 4 as an MILP model. This is shown in Fig. 9, in which the coefficients $A_{v u}^{i}, B_{v u}^{i}$, $C_{v u}^{i}, D_{v u}^{i},(i=0,1)$, and $E_{v u}$ are given in Table 2.

Table 2. Values of coefficients

\begin{tabular}{|c|c|c|c|}
\hline & $0 \leq \alpha_{v u}<\frac{\theta_{v}}{2}$ & $\frac{\theta_{v}}{2} \leq \alpha_{v u}<2 \pi-\frac{\theta_{v}}{2}$ & $2 \pi-\frac{\theta_{v}}{2} \leq \alpha_{v u}<2 \pi$ \\
\hline$A^{1}{ }_{v u}$ & $-\frac{1}{2 \alpha_{v u}+\theta_{v}}$ & $\frac{2}{4 \pi-2 \alpha_{v u}+\theta_{v}}$ & $-\frac{1}{2 \alpha_{v u}+\theta_{v}-4 \pi}$ \\
\hline$A_{v u}^{2}$ & $\frac{2}{\theta_{v}-2 \alpha_{v u}}$ & $-\frac{2}{2 \alpha_{v u}+\theta_{v}}$ & $\frac{2}{4 \pi+\theta_{v}-2 \alpha_{v u}}$ \\
\hline$B_{v u}^{1}$ & $\frac{1}{2}$ & $-\frac{2 \alpha_{v u}-\theta_{v}}{4 \pi-2 \alpha_{v u}+\theta_{v}}$ & $\frac{1}{2}$ \\
\hline$B_{v u}^{2}$ & $-\frac{4 \pi+2 \alpha_{v u}-\theta_{v}}{\theta_{v}-2 \alpha_{v u}}$ & $\frac{1}{2}$ & $-\frac{2 \alpha_{v u}-\theta_{v}}{4 \pi+\theta_{v}-2 \alpha_{v u}}$ \\
\hline$C_{v u}^{1}$ & $-\frac{2}{4 \pi-2 \alpha_{v u}-\theta_{v}}$ & $\frac{2}{2 \alpha_{v u}-\theta_{v}}$ & $-\frac{2}{8 \pi-2 \alpha_{v u}-\theta_{v}}$ \\
\hline$C_{v u}^{2}$ & $\frac{2}{4 \pi+2 \alpha_{v u}-\theta_{v}}$ & $-\frac{2}{4 \pi-2 \alpha_{v u}-\theta_{v}}$ & $\frac{2}{2 \alpha_{v u}-\theta_{v}}$ \\
\hline$D_{v u}^{1}$ & $\frac{4 \pi}{4 \pi-2 \alpha_{v u}-\theta_{v}}$ & 0 & $\frac{4 \pi}{8 \pi-2 \alpha_{v u}-\theta_{v}}$ \\
\hline$D_{v u}^{2}$ & 0 & $\frac{4 \pi}{4 \pi-2 \alpha_{v u}-\theta_{v}}$ & 0 \\
\hline$E_{v u}$ & 0 & -1 & 0 \\
\hline
\end{tabular}

In this formulation, $Z_{v u}, c^{1}{ }_{v u}$, and ${c^{2}}^{2}$ are integer variables; $P_{v}, F_{v u}$, and $\varphi_{v}$ are continuous variables. Note that $c_{v u}$ helps to simplify the form of constraints, but they are not independent variables. The number of variables in the formulation is approximately $4 n^{2}+2 n$, and the number of constraints is of the order of $O\left(n^{2}\right)$.

Recall that in Lemmas 1 to 4, we proved that any solution, which satisfies the constraints in (37) to (50), should be a multicast tree. In order to prove that the formulation of Problem MEM solves the minimum-energy multicast routing problem, it remains to show that every multicast tree can be expressed by the variables $P_{v}, Z_{v u}$,
$F_{v u}, c^{1}{ }_{v u}, c^{2}{ }_{v u}$ and $\varphi_{v}$ in Problem MEM. This is achieved by the following theorem.

Theorem 1. The formulation of Problem MEM solves the minimum-energy multicast problem.

Proof: Assume that $T_{s}=\left(N^{\prime}, A^{\prime}, q\right)$ is an arbitrary multicast tree of $G$. The solution corresponding to $T_{s}$ is obtained by setting variables in the following order:

1) If $(v, u) \in A^{\prime}$ then we set $Z_{v u}=1$;

2) We set all other $Z_{v u}=0$ otherwise;

3) If $v$ is an internal node of $T_{s}$, we set $P_{v}=\underset{u:(v, u) \in A^{\prime}}{\operatorname{Max}} p_{v u}$;

4) We set all other $P_{v}=0$ otherwise;

5) If $Z_{v u}=0$, we set $F_{v u}=0$;

6) If $Z_{v u}$ is not equal to zero, the corresponding $F_{v u}$ are assigned the value of the cardinality of $T_{s}^{u}$, i.e., $F_{v u}=\left|T_{s}^{u}\right|$, where $T_{s}^{u}$ is the set of nodes that belong to the sub-tree that contains node $u$ and is formed by removing $\operatorname{arc}(v, u)$ from the tree $T_{s}$, and it is clear that $\left|T_{s}^{u}\right|<n$;

7) We set $\varphi_{v}$ to be any direction such that the antenna beam of node $v$ can cover all the nodes $\left\{u \mid Z_{v u}=1\right\}$;

8) We set $c^{1}{ }_{v u}$ by Equations (14), (21), or (28) corresponding to different value of $\alpha_{v u}$;

9) We set $c^{2}{ }_{v u}$ by Equations (15), (22), or (29) corresponding to different value of $\alpha_{v u}$.

It is a straightforward exercise to show that those $P_{v}, Z_{v u}, F_{v u}, c^{1}{ }_{v u}$, $c^{2}{ }_{v u}$ and $\varphi_{v}$ values above satisfy the RTP, WMAP, AOP and Integrality Property constraints in Problem MEM.

Our analytical model can be easily applied in some specialized cases. Broadcast can be considered as a special case of multicast when $M=N$. Therefore, the constraints (39) and (40) in the formulation (see Fig. 9) disappear, and the constraint (41) can be simplified as constraints (51).

$$
\sum_{v \in N} F_{v u}-\sum_{v \in N} F_{u v}=1 ; \forall u \in N \backslash\{s\}
$$

Omni-directional antenna scenarios can also be considered as a special case of directional antenna model when the optimized variable $\varphi_{u}$ for each node $u$ is set to a constant $2 \pi$. Variables $\varphi_{u}$, $c_{v u}, c^{1}{ }_{v u}$, and $c^{2}{ }_{v u}$, and constraints $(44)-(49)$ all disappear, since $c_{v u}=1$ is always true.

\section{CONCLUSION}

In this paper we present a constraint formulation for the joint minimum-energy multicast and antenna orientation optimization problem in multi-hop ad hoc wireless networks. Based on the analysis on the properties of minimum energy multicast tree, the problem can be characterized in a form of mixed integer linear programming problem, and we proceed to prove the correctness of this formulation. To our best knowledge, these are the first work using integer programming to formulate the problem in a context of directional antenna applications. Many application scenarios can be solved efficiently based on the formulation using branch-and cut or cutting planes techniques. The optimal solutions can be used to assess the performance of heuristic algorithms for mobile networks by running them at discrete time instances. 
Objective function: minimize $\sum_{u \in N} P_{u}$

Subject to:

(I) Rooted Tree Property

$$
\begin{aligned}
& \sum_{v \in N} Z_{v s}=0 ; \\
& \sum_{v \in N} Z_{v u}=1 ; \forall u \in M \backslash\{s\} \\
& \sum_{v \in N} Z_{v u} \leq 1 ; \forall u \in N \backslash M \\
& \sum_{v \in N} Z_{u v} \leq(n-1) \sum_{v \in N} Z_{v u} ; \forall u \in N \backslash M \\
& \sum_{v \in N} F_{v u}-\sum_{v \in N} F_{u v}=\sum_{v \in N} Z_{v u} ; \forall u \in N \backslash\{s\} \\
& Z_{v u} \leq F_{v u} \leq(n-1) Z_{v u} ; \forall u \in N \backslash\{s\}, v \in N
\end{aligned}
$$

(II) Wireless Multicast Advantage Property

(III) Antenna Orientation Property

$$
\begin{aligned}
& c_{v u}^{1}>A_{v u}^{1} \varphi_{v}+B_{v u}^{1} ; \forall v, u \in N \\
& c_{v u}^{1} \leq C_{v u}^{1} \varphi_{v}+D_{v u}^{1} ; \forall v, u \in N \\
& c_{v u}^{2}>A_{v u}^{2} \varphi_{v}+B_{v u}^{2} ; \forall v, u \in N \\
& c_{v u}^{2} \leq C_{v u}^{2} \varphi_{v}+D_{v u}^{2} ; \forall v, u \in N \\
& Z_{v u} \leq c_{v u}^{1}+c_{v u}^{2}+E_{v u} ; \forall v, u \in N \\
& 0 \leq \varphi_{u}<2 \pi, \forall u \in N
\end{aligned}
$$

(IV) Integrality Property

$$
Z_{v u} \in\{0,1\} ; c_{v u}^{1} \in\{0,1\} ; c_{v u}^{2} \in\{0,1\}, \forall v, u \in N
$$

Figure 9. MILP model for Problem MEM

A major challenge, and a topic of continued research, is to extend our analytical model to large-scale networks with thousands of nodes. A near optimal solution can be found in a polynomial time using the Lagrange Relaxation and sub-gradient techniques [28] based on our formulation. Furthermore, it is important to develop the distributed algorithms of MEM to cope with the dynamic topologies.

\section{APPENDIX: NOTATION}

an arc set corresponding to the unidirectional wireless communication link;

$A^{\prime} \quad$ the arc set of multicast tree $T_{s}\left(N^{\prime}, A^{\prime}, q\right), A^{\prime} \subseteq A$;

$F_{v u}$ the nonnegative variables, which represent the amount of flow produced by the multicast initiator going through $(v$, $u)$; a directed graph modeling the wireless ad hoc network with a node set $N$, an $\operatorname{arc} \operatorname{set} A$, and an arc weight function $p$;

$M \quad$ a set of multicast members, $|M|=m$ and $M \subseteq N^{\prime}$;

$N \quad$ a node set in a two-dimensional plane, $|N|=n$;

$N^{\prime} \quad$ the node set of multicast tree $T_{s}\left(N^{\prime}, A^{\prime}, q\right)$ including all the multicast nodes $M \subseteq N^{\prime} \subseteq N$;
$P_{v} \quad$ the nonnegative continuous variables that represent the transmission power of the node $v$ required by the multicast tree $T_{s}$;

$T_{s} \quad$ a multicast tree of $G(N, A, p)$ rooted at a source node $s$;

$Z_{v u}$ the binary decision variables that are equal to one if arc $(v, u)$ exists in the sub-graph $T_{s}$ of $G$, and zero otherwise;

$c_{v u} \quad$ the binary variables such that $c_{v u}=1$ if node $u$ is covered by the antenna beam of node $v$ and 0 otherwise;

$p_{\text {max }}^{v}$ the maximum power level that node $v$ can choose;

$p_{v u} \quad$ a weight function $p: A \rightarrow R^{+}$that presents the power needed for the link from node $v$ to node $u$, which is always greater than zero;

$q_{v} \quad$ a weight (power) function $q: N^{\prime} \rightarrow R^{+} \cup\{0\}$ that can be zero;

$r_{v u} \quad$ the distance between node $v$ and node $u$;

$(.)^{*} \quad$ an optimized solution;

$\alpha \quad$ the propagation loss parameter

$\alpha_{v u} \quad$ the angle measured counter-clockwise from the horizontal axis to the vector from node $v$ pointing to node $u\left(0 \leq \alpha_{v u}\right.$ $<2 \pi)$;

$\theta_{v} \quad$ the beamwidth of node $v$ 's antenna $\left(0 \leq \theta_{v}<2 \pi\right)$; 
$\varphi_{v} \quad$ the antenna orientation $\left(0 \leq \varphi_{v}<2 \pi\right)$ of node $v$ defined as the angle measured counter-clockwise from the horizontal axis to the antenna boresight.

\section{REFERENCES}

[1] C. Diot, W. Dabbous, and J. Crowcroft, "Multipoint communication: a survey of protocols, functions, and mechanisms," IEEE Journal on Selected Areas in Communications, 15, pp. 277-290, April 1997.

[2] M. H. Ammar, G. C. Polyzos, and S. K. Tripathi, "Special issue on network support for multipoint communication," IEEE Journal on Selected Areas in Communications, 15, April 1997.

[3] J. E. Wieselthier, G. D. Nguyen, et al, "On the Construction of Energy-Efficient Broadcast and Multicast Trees in Wireless Networks", IEEE INFOCOM 2000, Conference on Computer Communications, March 2000, pp.585-594.

[4] J. H. Ryu and D. H. Cho, "A New Routing Scheme Concerning Power-Saving in Mobile Ad-Hoc Networks", Proceedings of IEEE International Conference on Communications, 2000, vol. 3, pp. 1719-1722.

[5] S. Singh, M. Woo, and C. S. Raghavendra, "Power-Aware Routing in Mobile Ad Hoc Networks", Proceedings of the International Conference on Mobile Computing and Networking, 1998, pp. 181-190.

[6] I. Stojmenovic and X. Lin, "Power-aware Localized Routing in Wireless Networks", Proceedings of IEEE International Parallel and Distributed Processing Symposium, 2000, pp. $371-376$.

[7] A. Clementi, P. Crescenzi, and et al, "On the Complexity of Computing Minimum Energy Consumption Broadcast Subgraphs", Proceedings 18th Annual Symposium on Theoretical Aspects of Computer Science, volume 2010 of LNCS, 2001, pp. 121-131.

[8] Song Guo and Oliver Yang, "Minimum-Energy Broadcast Routing in Wireless Multihop Networks", in Proceedings of IEEE Performance, Computing, and Communications Conference, Phoenix, Arizona, April 9 - 11, 2003, pp. 273 280.

[9] J. E. Wieselthier, G. D. Nguyen, and A. Ephremides, "Energy-Aware Wireless Networking with Directional Antennas: The Case of Session-Based Broadcasting and Multicasting", IEEE Transactions on Mobile Computing, July-September 2002 (Vol. 1, No. 3), pp. 176-191.

[10] J. E. Wieselthier, G. D. Nguyen, et al, "Energy-Limited Wireless Networking with Directional Antennas: The Case of Session-Based Multicasting", IEEE INFOCOM 2002, Conference on Computer Communications, March 2002.

[11] Akis Spyropoulos and C.S. Raghavendra, "Energy Efficient Communications in Ad Hoc Networks Using Directional Antennas", IEEE INFOCOM 2002, Conference on Computer Communications, March 2002.

[12] J. Chang and L. Tassiulas, "Energy Conserving Routing in Wireless Ad-hoc Networks", in Proceedings of IEEE INFOCOM 2000, March 2000.

[13] Y. Xu, J. Heidemann, and D. Estrin, "Geography-informed energy conservation for ad hoc routing", in Proceedings of MOBICOM 2001, Rome, July 2001.
[14] Q. Li, J. Aslam, and D. Rus, "Online power-aware routing in wireless ad-hoc networks", in Proceedings of MOBICOM 2001, Rome, Italy, July 2001.

[15] V. Rodoplu and T. H. Meng, "Minimum energy mobile wireless networks", IEEE Journal on Selected Areas in Communications, 17(8), August 1999.

[16] O. Egecioglu and T.Gonzalez, "Minimum-energy broadcast in simple graphs with limited node power", in Proceedings of IASTED International Conference on Parallel and Distributed Computing and Systems (PDCS 2001), pp.334338, Anaheim, CA, August 2001.

[17] F. Li and I. Nikolaidis, "On minimum-energy broadcasting in all-wireless networks", in Proceedings of the 26th Annual IEEE Conference on Local Computer Networks (LCN 2001), Tampa, Florida, November 2001.

[18] S. Singh, C. Raghavendra, and J. Stepanek, "Power-aware broadcasting in mobile ad hoc networks", in Proceedings of IEEE PIMRC, Osaka, Japan, September 1999.

[19] P. J. Wan, G. Calinescu, and et al, "Minimum-energy broadcast routing in static ad hoc wireless networks", in Proceedings of IEEE INFOCOM, Alaska, April 2001.

[20] J. E. Wieselthier, G. D. Nguyen, and A. Ephremides, "Energy-Efficient Broadcast and Multicast Trees in Wireless Networks", ACM Mobile Networks and Applications (MONET), 7(6): 481 - 492, December 2002.

[21] Y. B. Ko, V. Shankarkumar, and N. H. Vaidya, "Medium access control protocols using directional antennas in ad-hoc networks", in Proceedings of IEEE INFOCOM, March 2000.

[22] A. Nasipuri, S. Ye, J. You, and R. E. Hiromoto, "A MAC protocol for mobile ad hoc networks using directional antennas", in Proceedings of IEEE Wireless Communications and Networking Conference (WCNC), 2000.

[23] IEEE Local and Metropolitan Area Network Standards Committee, Wireless LAN medium access control (MAC) and physical layer (PHY) specifications, IEEE standard 802.11-1999.

[24] R. Ramanathan, "On the Performance of Ad Hoc Networks with Beamforming Antennas", in Proceedings of ACM Symposium on Mobile Ad hoc Networking and Computing (MobiHoc), 2001.

[25] A. Nasipuri, J. Mandava, H. Manchala, and R. E. Hiromoto, "On-demand routing using directional antennas in mobile ad hoc networks", in Proceedings of IEEE Computer Communications and Networks, 2000.

[26] D. Johnson, D. Maltz, Yih-Chun Hu, and Jorjeta G. Jetcheva, "Dynamic Source Routing”, Internet Draft, draft-ietf-manetdsr-06.txt, November 2001.

[27] C. Perkins, E. Belding-Royer, and S. Das, "Ad Hoc On Demand Distance Vector (AODV) Routing”, IETF Internet draft, draft-ietf-manet-aodv-09.txt, November 2001.

[28] D. P. Bertsekas, Nonlinear Programming, Athena Scientific, 1999. 